\title{
The expression of EMP1 is downregulated in oral squamous cell carcinoma and possibly associated with tumour metastasis
}

\author{
Jun Zhang, ${ }^{1,2}$ Wei Cao, ${ }^{1,2}$ Oin Xu, ${ }^{1,2}$ Wan-tao Chen ${ }^{1,2}$
}

'Department of Oral and Maxillofacial Surgery, Shanghai Key Laboratory of Stomatology, the Ninth People's Hospital, Shanghai Jiao Tong University, School of Medicine, Shanghai, PR China

${ }^{2}$ Department of Oral Clinical Immunology, School of Stomatology, Shanghai Jiao Tong University, Shanghai, PR China

\section{Correspondence to}

Wan-tao Chen, Department of Oral and Maxillofacial Surgery, Shanghai Key Laboratory of Stomatology, Ninth People's Hospital, Shanghai Jiao Tong University School of Medicine, 639, Zhizaoju Road, Shanghai 200011, PR China; chenwanta02002@hotmail. com

Accepted 30 September 2010 Published Online First 27 October 2010

\section{ABSTRACT}

Aims To investigate the expression of EMP1 in oral squamous cell carcinoma (OSCC) tissues and its correlation with available clinical parameters of patients with OSCC.

Methods The mRNA levels of EMP1 were measured in 18 paired OSCC and corresponding adjacent normal tissues using RT-PCR. Another 45 pairs of OSCC samples were selected to detect the mRNA level of EMP1 using quantitative RT-PCR (qRT-PCR). The protein levels of EMP1 were also evaluated in 60 cases of patients with OSCC using immunohistochemical staining. The correlation between EMP1 expression and clinical parameters was analysed with non-parametric analysis. Results The results of RT-PCR and qRT-PCR showed that, compared with the paired normal tissues, the mRNA levels of EMP1 were significantly decreased in OSCC. The immunohistochemical results indicated that the EMP1 protein was also downregulated in OSCC $(p=0.031)$. Decreased expression of EMP1 was significantly correlated with clinical stage $(p=0.002)$ and lymph node metastasis $(p=0.044)$ of patients with OSCC. Meanwhile, there was a significant difference between OSCCs at early and advanced stages $(p=0.003)$, and between OSCCs with lymph node metastasis and no lymph node metastasis $(p=0.045)$, respectively.

Conclusions The results suggest that EMP1 may be a tumour suppressor and associated with lymph node metastasis in OSCC.

\section{INTRODUCTION}

Head and neck squamous cell carcinoma (HNSCC) is the sixth most common malignancy worldwide. ${ }^{1}$ In China, the age-specific and age-standardised incidence rates (ASR) (per 100 000) were 1.2\% and $0.8 \%$, respectively, in the male and female populations in $2005 .^{2}$ Over $90 \%$ of HNSCC are oral squamous cell carcinoma (OSCC), which is undertreated and understudied. ${ }^{3}$

The initial events that occur in carcinogenesis are molecular changes, which could be useful if detected before the onset of symptoms and morphological changes. ${ }^{4}$ High-throughput microarray technology allows tissues to be interrogated for identifying the expression of thousands of genes simultaneously, thus generating a gene-expression profile for any given cancer. More importantly, it may be an efficient way to uncover the clues to the specific molecular derangements that contribute to cancer pathogenesis, and the recognition of pathognomonic alterations in gene expression may provide a basis for improved diagnosis and molecular classification of cancers. ${ }^{5} 6$ Our previous study has shown that the mRNA level of EMP1 gene decreases significantly in HNSCC samples tested by cDNA microarray.

EMP1, also called CL-20, ${ }^{8} \mathrm{TMP}^{9}{ }^{9} \mathrm{~B}^{\mathrm{10}}$ and PAP, ${ }^{11}$ was first described by Talyor et al in 1995 and is found in the gastrointestinal tract, skin, lung and brain but not in liver. ${ }^{12}$ Jain et al also reported that EMP1 is expressed in the tissues of the gastrointestinal tract, oesophagus, larynx, lip, oral mucosa, pharyngeal mucosa, colonic epithelia and tongue, ${ }^{13}$ and is mapped to chromosome $12 \mathrm{p} 12 .{ }^{14}$ As a marker, EMP1 was employed to distinguish invasive ductal and lobular carcinomas in breast cancer $^{15}$ and has been identified as a surface biomarker whose expression correlated with gefitinib clinical resistance. ${ }^{13}$ Gnirke found that there was a correlation between EMP1 expression and metastatic properties of several human mammary carcinoma cell lines. ${ }^{16}$

However, the function of EMP1 in OSCC is not clear currently. For the first time, the expression of EMP1 gene in OSCC tissues and its correlation with clinical parameters were investigated in this study.

\section{MATERIALS AND METHODS}

\section{Tissue acquisition}

Samples from patients with OSCC undergoing definite treatment were obtained from January to April in 2009 in the Department of Oral and Maxillofacial Surgery, Ninth People's Hospital Shanghai Jiao Tong University School of Medicine. The samples for each patient included OSCC tissue and normal tissue. Normal tissues were collected from oral epithelia at the surgical margin from the patients' oral cavity after tumour resection. The samples were snap-frozen in liquid nitrogen at the time of resection and stored at $-80^{\circ} \mathrm{C}$ until use. All patients selected in this study provided informed consent in advance.

\section{RNA preparation and RT-PCR}

Total RNA was extracted from all OSCC and normal samples using TRIzol reagent (Invitrogen; Life Technologies, Gaithersburg, Maryland) following the manufacturer's protocol. Five hundred nanograms of total RNA was reversetranscribed using TakaRa Reverse Transcriptase Reagents (TakaRa) and following the manufacturer's protocol. Primers for EMP1 and $\beta$-actin ${ }^{17}$ as a reference for normalisation were as follows: EMP1 sense 5'-ATTGCCAATGTCTGGTTGGTTT, antisense $5^{\prime}$-AGAACGCCGATGATGAAGCT; $\beta$-actin 
sense 5' - ATCGTCCACCGCAAATGCTTCTA, antisense 5' - AG CCATGCCAATCTCATCTTGTT. EMP1 and $\beta$-actin products are $380 \mathrm{bp}$ and $118 \mathrm{bp}$, respectively. Products were amplified by PCR using one denaturing cycle of $5 \mathrm{~min}$ at $95^{\circ} \mathrm{C}$, then $95^{\circ} \mathrm{C}$ for $30 \mathrm{~s}, 58.8^{\circ} \mathrm{C}$ for $30 \mathrm{~s}, 72^{\circ} \mathrm{C}$ for $30 \mathrm{~s}$ for 35 cycles and one final extension of $8 \mathrm{~min}$ at $72^{\circ} \mathrm{C}$.

\section{Quantitative real-time RT-PCR}

Quantitative real-time RT-PCR (qRT-PCR) was performed with Thermal Cycler Dice Real Time System TP800 (TakaRa) according to the standard protocol of SYBR Premix ExTaq perfect real time system (TakaRa). Primers for EMP1 were as follows: sense 5'-CCCTCCTGGTCTTCGTGT, antisense 5'-GG AATAGCCGTGGTGATA. Primers for $\beta$-actin were the same as those for RT-PCR. Thermal cycling conditions were $95^{\circ} \mathrm{C}$ for $1 \mathrm{~min}, 95^{\circ} \mathrm{C}$ for $15 \mathrm{~s}$ and 40 cycles at $60^{\circ} \mathrm{C}$ for $1 \mathrm{~min}$.

Expression of EMP1 in OSCC samples and adjacent normal samples was quantified by measuring the fractional cycle number at which the amount of expression reached a fixed threshold $\left(\mathrm{C}_{\mathrm{t}}\right)$ and directly related to the amount of product. The housekeeping gene, $\beta$-actin, was used as an internal control to quantify the products of EMP1. The relative quantification was given by the $C_{t}$ values, determined for triplicate reactions for OSCC and adjacent normal samples for EMP1 and for $\beta$-actin. Triplicate $C_{t}$ values were averaged and the $\beta$-actin $C_{t}$ subtracted to obtain $\Delta \mathrm{C}_{\mathrm{t}}\left(\Delta \mathrm{C}_{\mathrm{t}}=\mathrm{C}_{\mathrm{t}}\right.$ (target gene in OSCC/ adjacent normal sample) $-\mathrm{C}_{\mathrm{t}}$ ( $\beta$-actin gene in OSCC/ adjacent normal sample) $) . \mathrm{C}_{\mathrm{t}}$ values were calculated for each OSCC and adjacent normal sample. Relative expression level was determined as $2^{-\Delta \Delta \mathrm{Ct}}$, where $\Delta \Delta \mathrm{C}_{\mathrm{t}}=\Delta \mathrm{C}_{\mathrm{t}}$ (OSCC sample) $-\Delta \mathrm{C}_{\mathrm{t}}$ (adjacent normal sample). $2^{-\Delta \Delta \mathrm{Ct}^{\prime}}$ indicates the fold change in OSCC samples relative to adjacent normal samples.

\section{Immunohistochemistry and scoring of protein staining}

Specimens were reviewed by an independent pathologist to confirm the OSCC diagnosis. Sixty representative tissue paraffin blocks were chosen and cut into $5 \mu \mathrm{m}$ thin sections for immunohistochemistry (IHC). For EMP1 staining, the paraffinembedded sections were incubated for $30 \mathrm{~min}$ at $68^{\circ} \mathrm{C}$, deparaffinised in xylenes three times for $10 \mathrm{~min}$ each. Hydrating sections were processed gradually through graded alcohols: washed in $100 \%$ ethanol once for $5 \mathrm{~s}$, then $95 \%$ ethanol twice for $5 \mathrm{~s}$ each, washed in distilled water for $1 \mathrm{~min}$ with stirring to aspirate excess liquid from sections. Thereafter, sections were blocked for 10 min with $\mathrm{H}_{2} \mathrm{O}_{2}$. Then, they were washed three times for $5 \mathrm{~min}$ each in phosphate-buffered saline (PBS). Polyclonal mouse antihuman EMP1 (Abnova, Taiwan) was applied to the sections at dilution of 1:200, incubated overnight at $4^{\circ} \mathrm{C}$. On the second day, the sections were placed for $1 \mathrm{~h}$ at room temperature and the secondary antibody was applied to the samples after washing again three times for 5 min each with $\mathrm{PBS}$. The bound primary antibody was visualised by incubating the samples for $5 \mathrm{~s}$ in diaminobenzidine tetrahydrochloride (DAB; Sigma, St Louis, Missouri) solution at 1:80 dilution. The sections were rinsed in distilled water for $5 \mathrm{~min}$, counterstained with Harris haematoxylin, dehydrated, cleared and mounted.

Normal epithelial and cancer cells with an obvious membranous and/or cytoplasmic staining were defined as positive immunostaining. The scores of percentage of positive immunostaining $(\mathrm{P})$ were $0(<10 \%), 1(10-25 \%), 2$ (26-50\%), 3 $(51-75 \%)$ and $4(76-100 \%)$, and the scores for intensity of staining (I) were determined as 0 , negative; 1 , light yellow colour (weak staining); 2, brown colour (moderate staining); and 3, dark brown colour (strong staining). The total scores $(S)$ were designated as $\mathrm{P} \times \mathrm{I}$ for each section. ${ }^{18}$ The immunostaining scores were analysed by two experienced pathologists blinded to the treatment group.

\section{Acquisition of clinical information}

Clinical information for 60 patients not including survival rate because of the time of treatment was retrieved from the medical records, and all identifier data were terminally coded to maintain patient anonymity. The disease stage was defined as stage I-IV, based on the status of primary sizes, lymph nodes and metastases.

\section{Statistical analysis}

The SPSS 17.0 software package was used for statistical analysis. The correlation was analysed using the Spearman correlative analysis. Comparisons between the relative expression value of EMP1 or the intensity of its expression in OSCC and adjacent epithelia were analysed using a Wilcoxon non-parametric test for two related samples. Comparisons of the intensity of EMP1 expression in OSCC, between genders, anatomical locations, lymph node metastasis, clinical stages and histological grades, repectively, were analysed with Mann-Whitney's non-parametric test for two or $\mathrm{K}$ independent samples. A $\mathrm{p}$ value of $<0.05$ is considered to be significantly different.

\section{RESULTS}

RT-PCR and quantitative RT-PCR analysis for expression of EMP1in OSCC and adjacent normal tissues

RT-PCR was performed in 18 pairs of OSCC and the corresponding adjacent normal tissues, to confirm the EMP1 expression in mRNA level. It was found that the EMP1 expression decreased significantly in OSCC tissues (figure 1). To further quantify and confirm the above findings, another 45 pairs of OSCC samples were selected to test EMP1 mRNA by qRT-PCR. The EMP1 mRNA level ratio was calculated by the relative expression value of each OSCC tissues divided by the relative expression value of each adjacent normal tissues. Using the cut-off value of 0.5 , the expression ratio was less than 0.5 in $84.4 \%(38 / 45)$ of patients (figure $2 \mathrm{~A})$. For the mRNA levels of EMP1, there was a significant difference between OSCC and adjacent normal tissues $(\mathrm{p}=0.000)$ (figure $2 \mathrm{~B})$. The results further confirmed that expression of EMP1 was significantly decreased in OSCC.

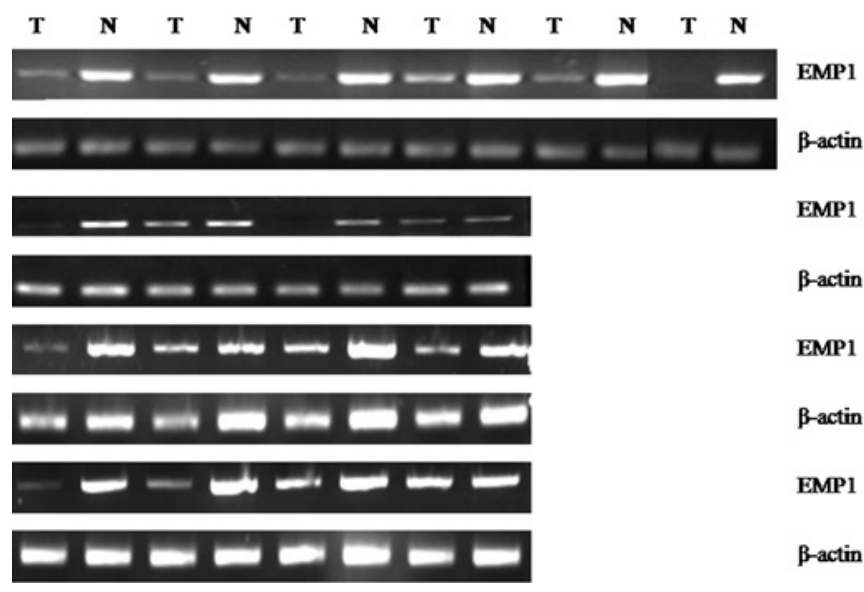

Figure 1 RT-PCR results of EMP1 in 18 pairs of samples. The EMP1 mRNA was decreased in most oral squamous cell carcinoma tissues compared with the corresponding adjacent normal tissues. 
Figure 2 Validation of the expression of EMP1 by qRT-PCR using another 45 pairs of samples. (A) Fold change in relative mRNA expression of EMP1 in samples from tumour and adjacent normal tissues. Dotted line, cut-off value. (B) Comparison for the relative expression of EMP1 between OSCC and adjacent normal tissues. Boxes, distribution of expression values from the 25th to 75th percentile for EMP1. Horizontal lines in boxes, median values; whiskers, 5th and 95th percentiles; dots, outliers; $p=0.000$.

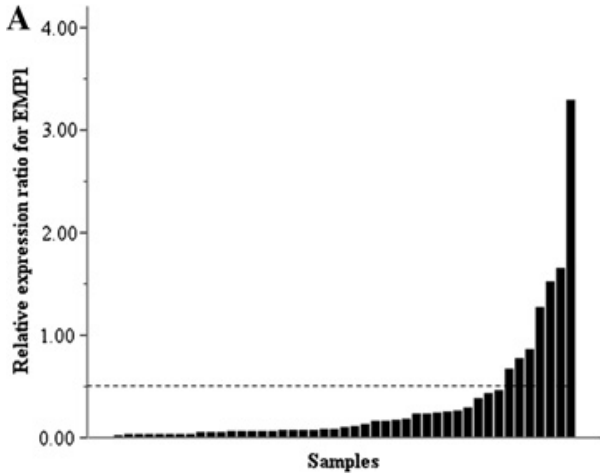

\section{Clinical data and immunohistochemical staining results}

Table 1 summarises the parameters of 60 patients with OSCC. The median age of the patients at the time of diagnosis was 61.7 (range 32-88) years. Moderate to strong staining was observed in $90 \%(27 / 30)$ of tumours with early stage and $71.4 \%$ (30/42) of tumours with no lymph node metastasis, repectively. In contrast, weak to moderate staining was observed in $50 \%$ $(15 / 30)$ of tumours with advanced stage and $55.6 \%(10 / 18)$ of tumours with lymph node metastasis, repectively. The correlations between lymph node metastasis, clinical stage and the EMP1 expression were indicated by a Spearman correlative analysis (table 2, $\mathrm{p}=0.044$ and $\mathrm{p}=0.002$, respectively). EMP1 expression was not correlated with gender, age, histological differentiation or primary sites.

To further validate EMP1 expression in protein level, we compared EMP1 in samples at early and advanced stages with or without lymph node metastasis via a Mann-Whitney non-parametric test (table $3, p=0.003$ and $p=0.045$, respectively). The results are shown in figure 3 .

Among these 60 sections, 39 cases had OSCC tissues and corresponding adjacent normal epithelia simutaneously in the same section, and the distance between the normal epithelia and

Table 1 Summary of demographic and clinical parameters $(n=60)$

\begin{tabular}{lcc}
\hline Variable & No & Percentage \\
\hline Age (mean \pm SD) & & - \\
Gender & $61.7 \pm 13.2$ & 40 \\
$\quad$ Female & 24 & 60 \\
$\quad$ Male & 36 & \\
Alcohol and tobacco & & 41.7 \\
$\quad$ Yes & 25 & 58.3 \\
$\quad$ No & 35 & \\
Anatomical locations & & 51.7 \\
$\quad$ Tongue & 31 & 23.3 \\
Cheek & 14 & 5 \\
$\quad$ Palate & 3 & 15 \\
$\quad$ Gingiva & 9 & 3.3 \\
Floor of mouth & 2 & 1.7 \\
$\quad$ Base of tongue & 1 & \\
Lymph node metastasis & & 30 \\
$\quad$ Yes & 18 & 70 \\
$\quad$ No & 42 & \\
Clinical stage & & \\
$\quad$ Early stage (I/II) & 30 & 50 \\
Advanced stage (III/IV) & 30 & 50 \\
Pathological grade & & \\
I II & 15 & 25 \\
III & 42 & 70 \\
\hline
\end{tabular}

OSCC tissues was more than $5 \mathrm{~mm}$ (figure $3 \mathrm{~A}$ ). We scored the EMP1 expression in two locations stated above. The average percentage of EMP1 positive immunostaining was $78 \%$ in normal epithelia but $67 \%$ in OSCC tissues. The results suggested that the average total scores of the expression levels of EMP1 in OSCC and in the adjacent normal epithelia were significantly different (table $3, p=0.031$ ) using the Wilcoxon non-parametric test, which further supported the previous findings obtained from the mRNA data.

\section{DISCUSSION}

At present, the effective treatment for OSCC is extensive surgical resection, along with chemotherapy and/or radiotherapy before or after surgery. In the past 30 years, the longterm survival rate for OSCC has not been improved, and only $60 \%$ of patients with OSCC survived for 5 years. ${ }^{19}$ Some novel biomarkers which reflect the physiological state and the change of cells during a disease process ${ }^{4}$ should be identified for designing individual therapy and predicting the prognosis for the patients with cancer. Therefore, ideal biomarkers can offer early and more accurate prediction and diagnosis for the patients with OSCC, particularly for an early-stage OSCC. In view of the potential use in the clinical practice for cancer biomarkers, a major focus in the cancer research is the identification of them in recent years. ${ }^{4} 20$

We have previously identified some different expressed genes in OSCC tissues compared with normal tissues using microarray. ${ }^{7}$ EMP1is one of the dysregulated genes, which has already been identified using a similar method in other studies, and its function might include controlling cell-cell interaction, cellular proliferation or regulating cellular differentiation..$^{51-23}$ Overexpression of EMP1 could inhibit proliferation of human oesophageal carcinoma cell line EC9706 transfected with the gene, among which the $\mathrm{S}$ phase was arrested and the G1 phase was prolonged. ${ }^{24}$ In this study, the decreased expression of EMP1 was qualitatively and quantitatively detected in mRNA level, in OSCC tissues compared with adjacent normal tissues,

Table 2 Correlation between clinical parameters of patients with oral squamous cell carcinoma and EMP1 expression levels

\begin{tabular}{lcl}
\hline Clinical characteristics & Correlation coefficient & p Value \\
\hline Gender & 0.023 & 0.860 \\
Alcohol and tobacco & 0.172 & 0.190 \\
Anatomical location & -0.043 & 0.743 \\
Lymph node metastasis & -0.261 & $0.044^{*}$ \\
Clinical stage & -0.384 & $0.002^{*}$ \\
Pathological grade & -0.072 & 0.586 \\
\hline${ }^{*} \mathrm{p}<0.05$. &
\end{tabular}


Table 3 Statistical analysis for the mean total scores of the expression levels of EMP1

\begin{tabular}{llll}
\hline & Mean total scores & $\mathbf{p ~ V a l u e ~}$ & $\mathbf{n}$ \\
\hline Adjacent normal tissue & $8.26 \pm 3.00$ & 0.031 & 39 \\
Tumour tissue & $7.21 \pm 3.02$ & & \\
Early stage & $8.63 \pm 2.50$ & 0.003 & 60 \\
Advanced stage & $6.57 \pm 3.01$ & & \\
Without lymph node metastasis & $8.05 \pm 3.06$ & 0.045 & 60 \\
With lymph node metastasis & $6.56 \pm 2.38$ & & \\
\hline
\end{tabular}

suggesting that EMP1 may be an OSCC suppressor in its initiation and development. Marvin et $a l^{8}$ observed that EMP1 mRNA and protein were induced during squamous differentiation of rabbit tracheal epithelial cells in vitro, and that EMP1 mRNA was most abundant in squamous epithelia as demonstrated by a Northern blot analysis and in situ hybridisation. It was also revealed that the protein expression for EMP1 was different in OSCC compared with adjacent epithelial cells by immunohistochemistry in this study, which indicated that the EMP1 expression at the protein level seems to be stronger in normal epithelial cells than in cancerous cells.

In addition, from an analysis of the gene-expression profile of oesophageal cancer cells after being transfected with EMP1 using the cDNA microarray, it was found that genes related to cell adhesion were upregulated, ${ }^{24}$ suggesting that EMP1 may play a main role in the process of SCC cells invasion and metastasis. In clinical practice, the choice of treatment modality for an individual patient needs to be carefully considered. Meanwhile, it is remarkable that attention must focus on choosing the most effective treatment while aiming to preserve organs and their function, and manage associated comorbid conditions. ${ }^{25}$ The traditional treatment planning and prognosis of OSCC are mainly based on TNM classification, but the TNM system is subjective and lacks predictive power for early OSCC, and provides little information regarding the aggressiveness of OSCC, prognosis and treatment response. ${ }^{26}$ It is widely accepted that a novel biomarker-based diagnosis assay can provide new approaches for early detection of cancer. The intensity of expression of EMP1, in this study, is correlated with lymph node metastasis and clinical stage, indicating that EMP1 may be involved in OSCC metastasis and its expression, which seems to provide some valuable information for choice of treatment or judgement of prognosis for clinicians combined with TNM classification.

It has been reported that EMP1 is downregulated in head and neck cancer, including oral cancer. ${ }^{5} \quad{ }^{21-23}$ However, reports about the function of EMP1 in OSCC are quite limited. To investigate whether EMP1 plays an important role in OSCC tumorigenesis, a validation of its expression levels in OSCC tissues and analysis of the correlation between its expression and clinical parameters were performed. This study has, for the first time, detected EMP1 expression in both mRNA and protein levels in OSCC by comparing OSCC with adjacent normal tissues and revealed the correlation between its expression levels and patients' clinical parameters.

In conclusion, the results suggest that EMP1 is downregulated and works as a tumour suppressor in OSCC. A further prospective study is needed to determine the value of EMP1 as a candidate biomarker and also to evaluate the usefulness as a survival factor for OSCC. Furthermore, experiments to determine the effect of the expression of EMP1 on cellular biological behaviour in vitro are necessary to understand the function of EMP1 in OSCC. The findings of our study provide the preconditions for a further study of the mechanism related to carcinogenesis in OSCC.
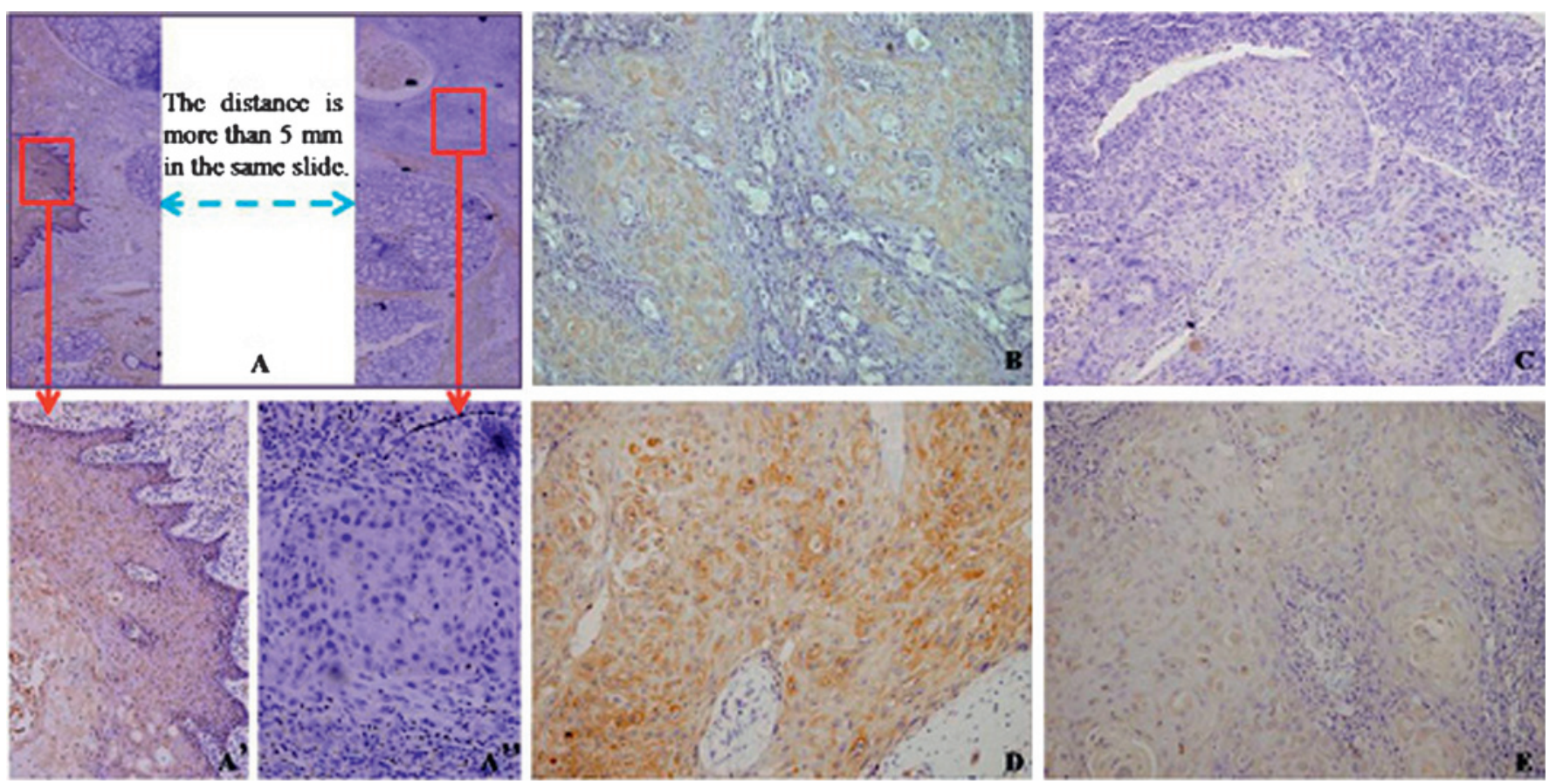

Figure 3 Representative sections in different samples. (A) Immunohistochemistrical staining of EMP1 in a section with corresponding adjacent normal epithelia and oral squamous cell carcinoma (OSCC) tissues (magnification $\times 100)$. $A^{\prime}$, staining in the adjacent normal epithelia (magnification of the above box); $A^{\prime \prime}$, staining in the OSCC (magnification of the above box). (B, C) EMP1 protein staining in OSCC with different stages (magnification $\times 200$ ). (B) OSCC with early stage (T2NOMO); (C) OSCC with advanced stage (T4NOMO). (D, E) EMP1 protein staining in OSCC without (D) or with (E) lymph node metastasis (magnification $\times 200$ ). 


\section{Take-home messages}

- The mRNA expression levels of EMP1 were detected by RT-PCR using 18 pairs of OSCC samples and by qRT-PCR using another 45 pairs of samples.

- The protein levels of EMP1 were evaluated by immunohistochemistry in 60 cases of samples, and its correlation with clinical parameters of patients with OSCC was analysed.

- The EMP1 expression at mRNA level was significantly decreased in OSCC tissues compared with corresponding adjacent normal tissues.

- Decreased expression of EMP1 protein was correlated with clinical stage and lymph node metastasis of patients with OSCC.

Acknowledgements The authors would like to express sincere thanks to all the staff in the Department of Oral Pathology for their help, especially to $L$ Wang, for her work in reviewing the original H\&E sections and evaluating the EMP1 staining, and to $\mathrm{J} \mathrm{Li}$, for evaluating the staining scores.

Funding This study was supported by National Natural Science Foundation of China (Grant No 30973343), Research Found of Shanghai Science and Technology Commission (08JC1414400, 09431902200, 10XD1402500) and Shanghai Leading Academic Discipline Project (S30206).

Competing interests None.

Patient consent Obtained.

Provenance and peer review Not commissioned; externally peer reviewed.

\section{REFERENCES}

1. Parkin DM, Bray F, Ferlay J, et al. Global cancer statistics, 2002. CA Cancer J Clin 2005:55:74-108.

2. Yang L, Parkin DM, Ferlay J, et al. Estimates of cancer incidence in China for 2000 and projections for 2005. Cancer Epidemiol Biomarkers Prev 2005;14:243-50.

3. Liu X, Chen Z, Yu J, et al. MicroRNA profiling and head and neck cancer. Comp Funct Genomics 2009. http://www.hindawi.com/journals/cfg/2009/837514.html.

4. Srinivas PR, Kramer BS, Srivastava S. Trends in biomarker research for cancer detection. Lancet Oncol 2001;2:698-704.

5. Kornberg LJ, Villaret D, Popp M, et al. Gene expression profiling in squamous cell carcinoma of the oral cavity shows abnormalities in several signaling pathways. Laryngoscope 2005;115:690-8.
6. DeRisi J, Penland L, Brown P0, et al. Use of a cDNA microarray to analyse gene expression patterns in human cancer. Nat Genet 1996;14:457-60.

7. Kuriakose MA, Chen WT, He ZM, et al. Selection and validation of differentially expressed genes in head and neck cancer. Cell Mol Life Sci 2004:61:1372-83.

8. Marvin KW, Fujimoto W, Jetten AM. Identification and characterization of a nove squamous cell-associated gene related to PMP22. J Biol Chem 1995:270:28910-16.

9. Ben-Porath I, Benvenisty N. Characterization of a tumour-associated gene, a member of a novel family of genes encoding membrane glycoproteins. Gene 1996; 183:69-75

10. Ruegg CL, Wu HY, Fagnoni FF, et al. B4B, a novel growth-arrest gene, is expressed by a subset of progenitor/pre-B lymphocytes negative for cytoplasmic mu-chain. $J$ Immunol 1996:157:72-80.

11. Schiemann S, Valentine $\mathrm{M}$, Weidle UH. Assignment of the human progression associated protein (PAP) to chromosome 12p12.3. Anticancer Res 1997;17:4281-5.

12. Taylor V, Welcher AA, Program AE, et al. Epithelial membrane protein-1, peripheral myelin protein 22, and lens membrane protein 20 define a novel gene family. $J$ Biol Chem 1995;270:28824-33.

13. Jain A, Tindell CA, Laux I, et al. Epithelial membrane protein-1 is a biomarker of gefitinib resistance. Proc Natl Acad Sci U S A 2005;102:11858-63.

14. Chen Y, Medvedev A, Ruzanov P, et al. cDNA cloning, genomic structure, and chromosome mapping of the human epithelial membrane protein CL-20 gene (EMP1), a member of the PMP22 family. Genomics 1997;41:40-8.

15. Turashvili G, Bouchal J, Baumforth $\mathrm{K}$, et al. Novel markers for differentiation of lobular and ductal invasive breast carcinomas by laser microdissection and microarray analysis. BMC Cancer 2007;7:55. http://www.biomedcentral.com/ $1471-2407 / 7 / 55$

16. Gnirke AU, Weidle UH. Investigation of prevalence and regulation of expression of progression associated protein (PAP). Anticancer Res 1998:18:4363-9.

17. Kajiro M, Hirota R, Nakajima Y, et al. The ubiquitin ligase CHIP acts as an upstream regulator of oncogenic pathways. Nat Cell Biol 2009;11:312-19.

18. Sarbia M, Loberg C, Wolter M, et al. Expression of Bcl-2 and amplification of c-myc are frequent in basaloid squamous cell carcinomas of the esophagus. Am J Pathol 1999:155:1027-32.

19. Coleman MP, Rachet B, Woods LM, et al. Trends and socioeconomic inequalities in cancer survival in England and Wales up to 2001. Br J Cancer 2004;90:1367-73.

20. Jain KK. Cancer biomarkers: current issues and future directions. Curr Opin Mol Ther 2007:9:563-71.

21. Alevizos I, Mahadevappa $M$, Zhang $X$, et al. Oral cancer in vivo gene expression profiling assisted by laser capture microdissection and microarray analysis. Oncogene 2001;20:6196-204.

22. Choi $\mathbf{P}$, Chen $\mathbf{C}$. Genetic expression profiles and biologic pathway alterations in head and neck squamous cell carcinoma. Cancer 2005:104:1113-28.

23. Cheong SC, Chandramouli GV, Saleh A, et al. Gene expression in human oral squamous cell carcinoma is influenced by risk factor exposure. Oral Oncol 2009:45:712-19.

24. Wang HT, Kong JP, Ding F, et al. Analysis of gene expression profile induced by EMP-1 in esophageal cancer cells using cDNA Microarray. World J Gastroenterol 2003:9:392-8

25. Choong N, Vokes E. Expanding role of the medical oncologist in the management of head and neck cancer. CA Cancer J Clin 2008:58:32-53.

26. Brinkman BM, Wong DT. Disease mechanism and biomarkers of oral squamous cell carcinoma. Curr Opin Oncol 2006;18:228-33. 